\title{
A 32 Year Old Man With Loss of Consciousness
}

\author{
Dana Critchell, $M D$
}

A 32-year-old man with no past medical history presented to the emergency department after losing consciousness at the end of a half-marathon. The patient was in his usual state of good health until the last mile of the marathon when he began to feel unsteady on his feet and short of breath. At the end of the race, he collapsed and was found unresponsive by EMS. The patient had no memory of the subsequent events and could only recall waking up in the emergency department feeling "numb" and "confused" several hours later. The patient denied any palpitations, chest pain, nausea, blurry vision, dizziness, urinary incontinence, or headache before or after the event. Of note, he was running outside approximately 3 to 4 times a week prior to this episode.

The temperature was $99.1^{\circ} \mathrm{F}\left(37.3^{\circ} \mathrm{C}\right)$, the pulse rate was 154 , and the blood pressure was $94 / 30$.

On physical examination, the patient had regained consciousness, but remained confused and disoriented with a GCS score of 13 . The patient was pale and diaphoretic, however he had moist mucous membranes and no evidence of skin tenting. His pupils were equal and reactive and the sclerae were anicteric. The heart, lungs, abdomen, and extremity examination were unremarkable. He had no costovertebral tenderness, lower extremity edema, or neck stiffness. Neurological exam revealed no focal deficits with intact cranial nerves. Several hours later, the patient was noted to be alert and oriented to person, place, and time. In the interim, the patient had several episodes of watery vomit.

Laboratory results on admission were notable for a normal CBC with a platelet count of $225 \mathrm{~B} / \mathrm{L}$ and a normal coagulation profile. The creatinine was $2.4 \mathrm{mg} / \mathrm{dL}$, the myoglobin was $303 \mathrm{ng} / \mathrm{mL}$, the CK was $157 \mathrm{IU}$, and the troponin was $0.15 \mathrm{ng} / \mathrm{mL}$. Electrocardiogram on admission revealed sinus tachycardia with an isolated ST elevation in AVR and ST depressions in II and V4V6. A repeat electrocardiogram showed resolution of these abnormalities. A chest x-ray and head CT scan showed no abnormalities. The patient was fluid resuscitated with normal saline at $200 \mathrm{cc} / \mathrm{hr}$ and admitted to the hospital with the diagnosis of heat exhaustion and rhabdomyolysis with acute renal failure.

During his hospital stay, the troponin peaked at $0.54 \mathrm{ng} / \mathrm{mL}$ and his creatinine dropped to $1.6 \mathrm{mg} / \mathrm{dL}$ with fluids resuscitation, and plans were made to discharge the patient the following morning. However, a $\mathrm{CBC}$ the following morning revealed marked thrombocytopenia with a platelet count of $64 \mathrm{~B} / \mathrm{L}$ and the CK levels were now significantly elevated at 7105 IU. The patient remained in the hospital to monitor his platelet count, which rose to $97 \mathrm{~B} / \mathrm{L}$ the following day. The patient was then discharged with instructions for a follow-up $\mathrm{CBC}$ with his primary care provider in the near future. Interestingly, another participant in the halfmarathon presented with acute renal failure followed by thrombocytopenia to $80 \mathrm{~B} / \mathrm{L}$ the day after presentation.

\section{Discussion}

Heat exhaustion represents only one part of a continuum of heatrelated illnesses, ranging from heat stress to heat exhaustion to heat stroke. While heat stress is simply perceived discomfort in a hot environment, typically during physical exertion, heat exhaustion is a more severe form of heat stress secondary to water or salt depletion from excessive heat. Patients start becoming symptomatic at this point, with many experiencing dizziness, weakness, extreme thirst, headache or syncope. More serious manifestations, such as delirium and seizures, are notably absent. Body temperatures can vary anywhere from 37 to $40^{\circ} \mathrm{C}$. Once core body temperatures rise above approximately $40^{\circ} \mathrm{C}$, patients begin to develop heat stroke, the most virulent form of the heat-related illnesses.

Heat stroke requires two findings for its diagnosis, namely a core body temperature greater than approximately $40{ }^{\circ} \mathrm{C}$ and central nervous system dysfunction, whether it be convulsions, coma, delirium, or simple confusion. ${ }^{1}$ While heat stress and exhaustion are typically considered relatively benign conditions, heat stroke is a true medical emergency with mortality rates ranging from $20 \%$ to $70 \%$ depending on patient age. Its pathogenesis and potential lethality are thought to be due to a combination of the direct cytotoxic effects of heat, the physiological demands this heat places on the body, and the host's inflammatory and coagulation response.

Two types of heat stroke are described, one being classic heat stroke and the other, exertional heat stroke. Classic heat stroke is typically seen in those at the extremes of age and also in those with underlying medical illnesses, including certain cardiovascular, neurological and psychiatric disorders. In addition, individuals on certain medications such as diuretics or anticholinergic agents are at an increased risk. Exertional heat stroke, on the other hand, often affects young, healthy individuals engaged in strenuous activity during times of high heat and humidity. Interestingly, testing on some of these patients reveals a susceptibility to malignant hyperthermia which may predispose them to the development of heat stroke.

Despite this patient's modest temperature elevation and moderate physical stressor given his history of regular physical exercise, this patient appeared to suffer from many of the more serious sequelae classically seen only with heat stroke, including a change in mental status, myocardial stress, rhabdomyolysis, acute renal failure and thrombocytopenia. Neurologic dysfunction, whether secondary to metabolic derangements, edema, infarction, or hypernatremia, is, by definition, seen in all cases of heat stroke. While some patients have gross neurological disturbances such as coma or encephalopathy, other patients present with more subtle signs such as inappropriate behavior or impairment in judgment.1 Unfortunately, patients often do not recover fully from these neurological insults. One study found that 33\% of patients who presented with heat stroke and survived during the 1995 Chicago 
heat wave suffered long-term moderate to severe functional neurological impairment.

Other potential complications of heat stroke involve virtually any organ system and can rapidly lead to multi-organ dysfunction and death even with proper supportive measures. Among these complications are acute respiratory distress syndrome, myocardial or hepatocellular injury, rhabdomyolysis, and acute renal failure. Also commonly seen in heat stroke are bleeding diatheses, most notably disseminated intravascular coagulation and isolated thrombocytopenia.

One proposed theory behind the phenomenon of DIC in heat stroke involves the splanchnic vasoconstriction and peripheral vasodilation that occurs as part of the body's compensatory mechanisms after exposure to extreme heat. This vasoconstriction can lead to intestinal ischemia and oxidative stress, resulting in loss of the protective gastrointestinal barrier and leakage of endotoxins into the systemic circulation. In addition, heat shock itself induces an acute-phase response to help protect the body from tissue injury. Inflammatory and anti-inflammatory cytokines, most notably interleukin-1 and 6, TNF-alpha and interferon-alpha, are elevated in heat shock, which contributes to the thermoregulatory failure and circulatory shock not only directly but also via increased production of nitric oxide which further promotes intestinal permeability. ${ }^{1}$

Researchers have found that thrombocytopenia can also develop in heat stroke in the absence of DIC. Furthermore, one case report detailed a patient very similar to our patient with heat exhaustion who presented with thrombocytopenia as well as leukopenia, both presumably secondary to thermolysis. Thus, the hemostatic changes in heat stroke are likely multifactorial in nature, a combination of DIC, thrombocytopenia independent of DIC, and failure of coagulation factor production secondary to hepatic injury.?

Treatment of heat stroke focuses on mainly on rapid cooling and support of organ systems. Various cooling techniques can be employed since no specific method has been shown to be superior. Common methods include whole body immersion or the use of fans to aid in conductive heat losses with cold water or ice to the skin. Regardless, achieving a rectal temperature of $39.4^{\circ} \mathrm{C}$ or less within thirty minutes of presentation has been shown to improve survival. As of yet, there appears to be no role for medications such as dantrolene, and the use of anti-pyretics has not been evaluated thus far. Unfortunately, prompt cooling may not prevent the development of tissue injury, patients should continue to be observed even after stabilization and resolution of hyperthermia. ${ }^{1}$

This case is unique in that despite his diagnosis of heat exhaustion, this patient developed several complications that typically occur with the more deadly form of heat-related illnesses, heat stroke. In addition, the dramatic fall in his platelet count highlights not only the potential virulence of heat stroke but also, its pathogenesis. In summary, due to a combination of heat toxicity itself and a breakdown of the gastrointestinal barrier with production of inflammatory cytokines, patients with heat stroke and even heat exhaustion are vulnerable to thrombocytopenia isolated from or in conjunction with DIC. As such, even after hyperthermia has resolved, one should remain vigilant of this potential complication, as well as many others, in all patients who present along the continuum of heat-related illnesses.

\section{References}

1. Bouchama A, Knochel J. Heat stroke. N Engl J Med June 2002; 246 (25): 1978-88.

2. Yeo, Theresa. Heat Stroke: A comprehensive review. AACN Clinical Issues, 2003; 15(2): 280-93.

3. Mechem, C Crawford. Severe Hyperthermia: heat stroke, neuroleptic malignant syndrome, and malignant hyperthermia. UpToDate online, April 2005.

4. Bendahan D, Kozak-Ribbens G, Confort-Gouny S. A noninvasive investigation of muscle energetics supports similarities between exertional heat stroke and malignant hyperthermia. Anesthesia \& Analgesia Sept 2001; 93(3): 683-689.

5. Dematte J, O'Mara K et al. Near-fatal heat stroke during the 1995 heat wave in Chicago. Ann Int Med Aug 1998; 129(3): 173-81.

6. Lambert, Patrick. Role of gastrointestinal permeability in exertional heat stroke. Exercise and Sports Sciences Review, Oct 2004; 32 (4): 185-90.

7. Mustafa K, Omer O, Khogali m et al. Blood coagulation and fibrinolysis in heat stroke. British J of Hematology 1985; 61: 517-23.

8. Lohiya S, Lohiya G, Humerez R. Heat exhaustion with thrombocytopenia and leukopenia in a car wash attendant. Am J of Hematology 1996; 52: 120-21.

9. Grogan H, Hopkins P. Heat Stroke: Implications for critical care and anesthesia. British Journal of Anaesthesia 2002; 88(5): 700-07.

10. Dematte J, O'Mara K et al. Near-fatal heat stroke during the 1995 heat wave in Chicago. Ann Int Med Aug 1998; 129(3): 173-81.

11. Bouchama A, Cafege A, Devol EB et al. Ineffectiveness of dantrolene sodium in the treatment of heatstroke. Crit Care Med Feb 1991: 19(2): 176-80. 